\title{
Eye Gaze in HMI to Design a Crane's UI
}

\author{
Jouh Yeong Chew* \\ University of Tokyo \\ Bunkyo-ku, 7-3-1 Hongo \\ jychew@delight.t.u-tokyo.ac.jp
}

\author{
Koichi Ohtomi \\ University of Tokyo \\ Bunkyo-ku, 7-3-1 Hongo \\ koichi.ohtomi@delight.t.u-tokyo.ac.jp
}

\author{
Hiromasa Suzuki \\ University of Tokyo \\ Bunkyo-ku, 7-3-1 Hongo \\ suzuki@den.t.u-tokyo.ac.jp
}

\begin{abstract}
Vision constitutes a significant part of information input for Human Machine Interaction (HMI), and the understanding of gaze characteristics such as stability, focus, and duration is promising to design a good User Interface (UI). This paper defines HMI events as UI design factors and identifies its correlation with users' feedback, i.e. Gaze Metrics (GM) and affect. The observation of the trilateral relationship between these parameters during a pilot testing offers insights to designers on how to improve $\mathrm{UI}$ design to enhance usability and attractiveness.
\end{abstract}

Keywords. Gaze fixation metric. Subjective bias. Psychophysiology. Delight design. Kano model.

\section{INTRODUCTION}

This study proposes a concept to use GM as the objective measure to bridge HMI with Delight Design, which is a design methodology to fulfil the 'excitement' of Kano model (Verduyn, 2014). Heo (2007) and Mayer (2012) implemented the Kano model to improve the design of UI. Self-reported measures such as questionnaires and Semantic Differential (SD) are commonly used to evaluate users' feedback. These methods are susceptible to bias such as social masking and cultural differences (Jang, 2015). Thus, an objective measure such as that of a physiological response is desirable.

Eye gaze is preferred because it is dominant in environment perception and decision making (Poitschke, 2011). Its contribution ranges from product designs (Kohler, 2015) to input modalities for intelligent systems (Biswas, 2015). Despite previous efforts, the relationship between UI design factors and gaze behaviour remains ambiguous.

This study addresses these gaps by mapping GM to UI design factors and making comparisons with the results of the conventional method using SD. As far as we understand, this trilateral relationship among UI design factors, psychology and physiology has yet to be studied. This concept (1) facilitates the evaluation of the feasibility to use GM to reduce subjective bias, and (2) reduces the ambiguity between UI design factors and users' response such as gaze behaviour and 'excitement'.

\section{EXPERIMENT SETUP AND METHODS}

A pilot testing was carried out on a simulator to improve cranes' UI. There were three users with eight trials each. The task was to pick up the load, avoiding two obstacles and unload it at another position. It was difficult because of depth perception when moving the load in the radial direction. The design factors were (a) the Cartesian coordinates of the UI with respect to the operator's view, and (b) the addition of the 'topview', which showed the bird's eye view of the operating environment. The objectives were to evaluate the optimal position of the $\mathrm{UI}$ and the feasibility of adding the 'topview'.

Gaze behaviour was recorded simultaneously during the operation at $120[\mathrm{~Hz}]$. The fixed-type Tobii Pro X3-120 was used because of its larger tolerance to head movements. Gaze fixations were mapped onto seven Area of Interests (AOls), which were the object being hoisted, its four neighbours, the 'topview' display, and fixations on non-relevant areas. The mapped gaze fixations were used to calculate the Markov transition model for each trial (Chew, 2015). Four GM were derived from the model - transition entropy $H_{t}$, stationary entropy $F_{s}$, matrix density $P_{\mathrm{m}}$ and the function of norm-2 of eigenvalues $\mathbf{D}$. A stable gaze was defined by a small $H_{z}$ and a long gaze duration was defined by a small $\mathbf{D}$. Gaze focus was defined by small $H_{s}$ and $\rho_{\mathrm{m}}$. The linear combination of these GM defines a gaze pattern. For instance, a small magnitude defines a 'stable gaze with focus'.

Affective adjectives with 7-point Likert scale were used to evaluate the users' 'excitement'. Steps were taken to reduce variability. Users were given a list of 14 adjectives and were asked to identify adjectives which they understood. The unanimously understood set yielded six adjectives - 'convenient', 'clear', 'comfort', 'satisfied', 'relax', and 'easy', which were used for further evaluation in the next section. 

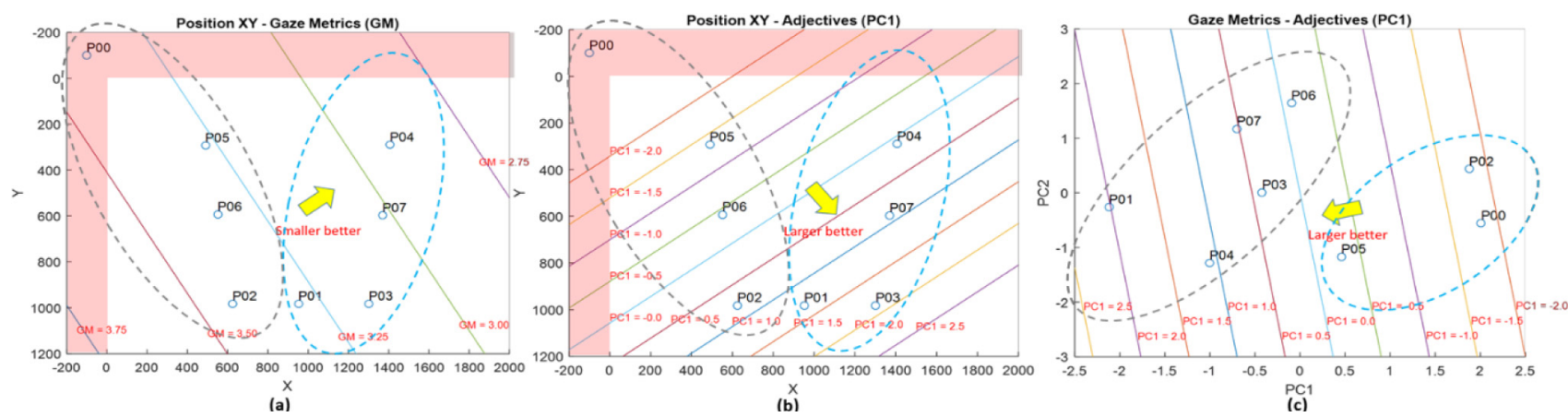

Figure 1: (a) Mapping of GM - design factors, (b) Mapping of 'excitement' - design factors, (c) Mapping of 'excitement' - GM

\section{PRELIMINARY RESULTS AND DISCUSSION}

Three design maps are used to recommend $\mathrm{UI}$ design in terms of its usability and attractiveness. Figure 1(a) maps GM to design factors. The $x$ - and $y$ - axes represent the Cartesian coordinates and the trials are plotted as 'P00' to 'P07'. The trial without 'topview' is defined in negative coordinates region in red. The correlation between $\mathrm{GM}$ and design factors is evaluated using Multiple Linear Regression (MLR). The dependent variable of the MLR model is rearranged to be the intercept of the linear equation and it is plotted as lines on Figure 1(a). Each line corresponds to different value of the dependent variable - GM. The results suggest (a) 'P04' is the most preferred and 'P02' is the least preferred because smaller GM corresponds to stable gaze with focus (Chew, 2015), (b) despite the linearity of MLR model, the Spearman's monotonic correlation between MLR estimation and actual value reasonably stood at 0.762 .

Figure 1(b) shows the correlation between design factors and the users' 'excitement', which is represented using PC1 of Principal Component Analysis (PCA) of the six adjectives. A comparison is made in Figure 1(a) and it suggests that (a) 'P03' is the most preferred and 'P0O' is the least preferred. The results are not exactly the same but it is within expectations due to bias and linearity of MLR model. K-means clustering of the design factors into two groups provided further insights. Referring to Figure 1(a), 'P03' is in the preferred cluster and 'P0O' is in the less preferred one. Thus, despite bias and limitation of the model, the maps are able to provide a reference to the preferred region rather than the exact position. (b) The Spearman's correlation is 0.524 which indicates that result obtained using GM is more robust. This is possibly because of the absence of social masking and cultural difference, both of which usually affect SD method. Figure 2 shows the coefficient of variation for GM and adjectives, in which smaller variation is commonly evident for objective measures, especially the linear combination of GM.
The third design map shows the relationship between GM and the users' 'excitement'. The Spearman's correlation is poor at 0.429 . However, it is worth noting a general pattern that shows a correspondence between smaller GM and the users' 'excitement'. This is true for extreme cases such as the best-performing and worst-performing cases. In general, the results suggest that (a) the optimal position of $\mathrm{UI}$ is possibly on the right of the operator's view which consists of 'P04', 'P07' and 'P03', and (2) the addition of 'topview' is desirable because ' $\mathrm{P} 00$ ' is common among the least preferred design and this is consistent for design maps using GM and users' 'excitement'.

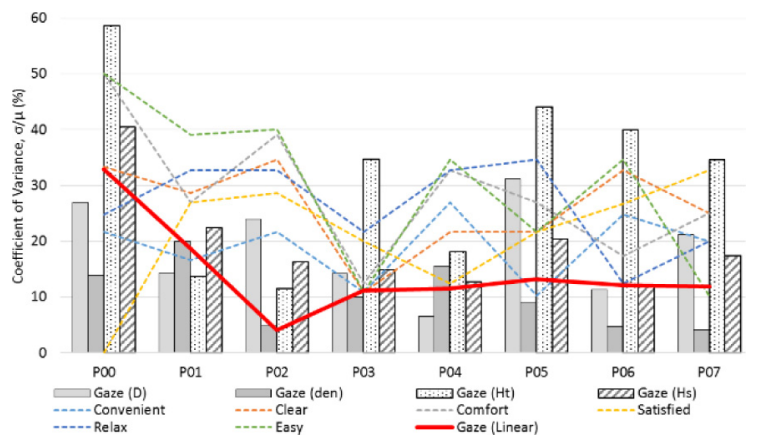

Figure 2: Comparison of coefficient of variance between objective and subjective measures

\section{CONCLUSIONS}

A concept to use GM for HMI analysis is proposed. The results suggest a possibility of using GM to reduce subjective bias. The correlation between design factors are also mapped onto $\mathrm{GM}$ and users' 'excitement'. However, MLR model is inadequate to represent nonlinearity of real-world problems. The pilot testing offers insights to designers on how to enhance the usability and attractiveness of UI. The results warrant further validation of its feasibility by increasing the sample size, addressing the limitation of MLR model, and executing multi-objective optimization of objective and subjective evaluations. 


\section{ACKNOWLEDGEMENT}

This research was supported by New Energy and Industrial Technology Development Organization (NEDO) of Japan, and we would like to thank them for their assistance.

\section{REFERENCES}

Biswas, P. and Langdon, P. (2015) Multimodal Intelligent Eye-Gaze Tracking System. Intl. J. Human-Computer Interaction, 31. 277-294.

Chew, J.Y., Ohtomi, K. and Suzuki, H. (2015) Quantitative Measure of Operator's Experience Based on Gaze Fixation: A Preliminary Study. In: Proceedings of A-DEW 2015. Hong Kong, 29-30 Oct. 146-150.

Heo, J., Park S. and Song, C. (2007) A Study on the Improving Product Usability Applying the Kano's Model of Customer Satisfaction. In: Jacko, J. (eds). Human-Computer Interaction, LNCS 4550. Heidelberg: Springer. 482-489.
Jang, E.H., Park, B.J., Park, M.S., Kim, S.H. and Sohn, J.H. (2015) Analysis of Physiological Signals for Recognition of Boredom, Pain, and Surprise Emotions. Journal of Physiological Anthropology, 34(25). 1-12.

Kohler, M., Falk, B. and Schmitt, R. (2015) Applying Eye-Tracking in Kansei Engineering Methodology for Design Evaluations in Product Development. Intl. J. Affective Eng., 14. 241-251.

Mayer, J.H. (2012) Using the Kano Model to Identify Attractive User-Interface Software Components. In: Proceedings of ICIS2012. Florida, 16-19 Dec.

Poitschke, T., Laquai, F., Stamboliev, S. and Rigoll G. (2011) Gaze-Based Interaction on Multiple Displays in an Automotive Environment. In: Proceedings of the IEEE Intl. Conf. SMC 2011. Alaska, 9-12 Oct. 543-548.

Verduyn, D. (2014) Discovering the Kano Model. http://www.kanomodel.com/discovering-thekano-model/ (2016/5/10) 